\title{
Deregulation of microRNA expression in peripheral blood mononuclear cells from patients with $\mathrm{HCV}$-related malignancies
}

\author{
Alessia Piluso $^{1}$ - Laura Gragnani ${ }^{1}$ Elisa Fognani ${ }^{1} \cdot$ Elena Grandini $^{2}$. \\ Monica Monti ${ }^{1}$ - Cristina Stasi $^{1} \cdot$ Elisabetta Loggi $^{2} \cdot$ Marzia Margotti $^{2}$. \\ Fabio Conti ${ }^{2} \cdot$ Pietro Andreone $^{2} \cdot$ Anna Linda Zignego $^{1}$
}

Received: 23 March 2015 / Accepted: 27 July 2015/Published online: 14 August 2015

(C) Asian Pacific Association for the Study of the Liver 2015

\begin{abstract}
Background and aim Hepatocellular carcinoma is one of the major causes of death due to cancer worldwide, and its association with hepatitis $\mathrm{C}$ virus infection has been definitively established. Hepatitis $\mathrm{C}$ virus is also involved in the pathogenesis of non-Hodgkin's lymphoma. This is the only virus infecting humans that is able to induce two different malignancies. We analyzed the expression levels of a panel of microRNA in peripheral blood mononuclear cells of patients with hepatitis $C$ virus-related malignancies in order to find a disease-associated deregulation and identify specific biomarkers.

Methods We tested peripheral blood mononuclear cells isolated from patients with hepatocellular carcinoma, nonHodgkin's lymphoma, hepatitis C virus without malignancies and healthy subjects for a panel of microRNA selected on the basis of previous studies. MicroRNA expression was evaluated by real-time PCR.
\end{abstract}

Alessia Piluso and Laura Gragnani contributed equally to this work.

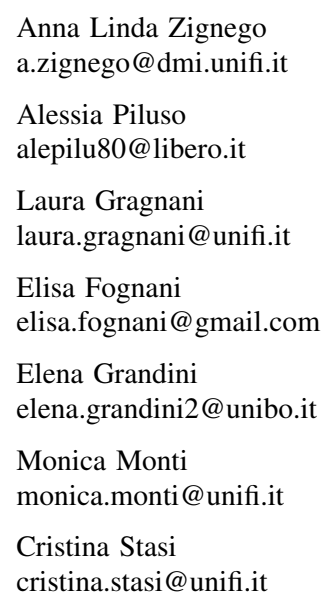

Results Our results showed an upregulation of miRNA21 and downregulation of miRNA-26b in hepatocellular carcinoma and non-Hodgkin's lymphoma patients compared to controls $(p<0.001)$. Deregulation of miRNA-16 and miRNA-155 was limited to lymphoma patients.

Conclusions This study shows that some microRNAs are differently expressed in peripheral blood mononuclear cells from hepatitis $\mathrm{C}$ virus patients who develop hepatocellular carcinoma or lymphoma, while others share a common behavior. Thus, analysis of the expression of microRNAs could be a noninvasive marker of hepatitis $\mathrm{C}$ virus-related carcinogenesis. This analysis could be a suitable tool for identifying the existence of a malignancy and also discriminating between these two hepatitis $\mathrm{C}$ virus-related cancers.

Keywords Hepatitis C virus - microRNA . Hepatocellular carcinoma · Non-Hodgkin's lymphoma . Biomarker

Elisabetta Loggi

elisabetta.loggi@unibo.it

Marzia Margotti

marzia.margotti2@unibo.it

Fabio Conti

fabio.conti2@studio.unibo.it

Pietro Andreone

pietro.andreone@unibo.it

1 Department of Experimental and Clinical Medicine, Center for Systemic Manifestations of Hepatitis Viruses (MASVE), University of Florence, Largo Brambilla 3, 50134 Florence, Italy

2 Department of Medical and Surgical Sciences, University of Bologna, Via Massarenti 9, 40138 Bologna, Italy 


\section{Introduction}

Hepatitis C virus (HCV) infection is a primary public health problem with about 200 million HCV carriers worldwide. Hepatocellular carcinoma (HCC) is a major malignancy worldwide, being the second most frequent cause of cancer death in males and the sixth in females [1]. $\mathrm{HCV}$ is responsible for $33 \%$ of $\mathrm{HCCs}$ in developing countries, and since no vaccine is available, the only strategy to implement is prevention [1]. HCV is also involved in the pathogenesis of non-Hodgkin's lymphoma (NHL) [2-4]. For these reasons, HCV is the only human virus able to induce two different kinds of cancer.

The evolution of HCC may occur from the progress of different stages of hepatitis and cirrhosis, while lymphoma results from overexpanded B cell clones characterizing mixed cryoglobulinemia (MC), a benign but prelymphomatous HCV-related lymphoproliferative disorder [57]. The exact mechanisms by which HCV induces both HCC and NHL remain undefined. Several reports have demonstrated that HCV alters the expression of specific oncogenes and tumor-suppressor genes implicated in carcinogenesis and/or lymphomagenesis [3, 4], and the host's genetic background also seems to play a role [8].

In the last 10 years, research interest has been focused on a class of epigenetic post-transcriptional regulators, the microRNAs (miRNAs). Acting on gene expression modulation, the miRNAs are involved in the fine tuning of physiological responses and make an important contribution to the pathogenesis of several diseases. Modifications in specific miRNA patterns are correlated with several types of human cancers and seem to play a role in different aspects of carcinogenesis [9].

Numerous studies are available today regarding HCC, several reporting unique profiles with only a few miRNAs in common, suggesting that the pathophysiology of HCC is complex and heterogeneous. So far, downregulation, mostly in HCV-negative HCCs, has been described for miR-122, a microRNA specific for the liver [10-12]. Also, less than normal levels of miR-199a/b-3p, of members of the miR-100 family (miR-100 and miR-99a) and of the let-7 family have been reported in HCCs of different etiology, although the opposite behavior was observed for let- $7 \mathrm{a}$ and let- $7 \mathrm{f}$ in viralnegative HCCs $[10,11,13]$. MiR-21 is reported as the most upregulated miRNA in HCC [14]: since one of its target genes is the tumor-suppressor PTEN (phosphatase and tensin homolog), miR-21 upregulation reflects lower PTEN expression, contributing to HCC growth, migration and invasion [14]. A recent paper has attributed to serum levels of miR-1 and miR-122 a predictive value for overall survival in HCC of different etiologies, showing the importance of miRNAs as biological markers [15].
Since miRNAs play a role in different stages of the hematopoietic maturation process, they are implicated in several blood malignancies and are correlated with prognosis and phenotype. Several studies have confirmed the involvement of cancer-related and newly identified miRNAs, namely miR-155, miR-146a, miR-21 and miR-26b, in the pathogenesis of different subtypes of lymphomas including some HCV-related ones (marginal zone lymphoma, MZL and diffuse large B cell lymphoma, DLBCL) [16-19]. MiR-21 and miR-26b have also been associated with the development of HCC and poor outcome after liver cancer therapy, respectively [20, 21]. Notably, downregulation of miR26b, previously shown in HCV-related NHL [16], has been confirmed in patients with $\mathrm{MC}$, suggesting its involvement also in this prelymphomatous condition [22].

However, the biological significance of miRNA deregulation in HCV-related HCC and lymphomas, together with targets and biochemical mechanisms, needs to be clarified. To date, there are no reliable diagnostics for predicting the risk that chronic $\mathrm{HCV}$ infection will evolve into $\mathrm{HCC}$ or NHL and, for this reason, there is increasing interest in translational research able to enhance our knowledge of this topic. In this light, in the present study we have evaluated the expression levels of a panel of selected miRNAs in peripheral blood mononuclear cells (PBMCs) of patients with HCV-related malignancies, HCC and NHL in order to clarify disease-associated deregulation and identify novel specific biomarkers.

\section{Patients and methods}

\section{Patients}

We studied $125 \mathrm{HCV}$ chronically infected patients with the following characteristics: 85 with $\mathrm{HCV}$-positive chronic hepatitis (HCV), 15 with HCV-associated NHL (NHL$\mathrm{HCV}$ ), 25 with $\mathrm{HCV}$ positive HCC (HCC-HCV) and 40 healthy blood donors (HS) as negative controls, referred to the outpatient clinics of the MASVE Center of the University of Florence and of the Department of Medical and Surgical Sciences of the University of Bologna. The main clinical, serological and virological data of subjects are reported in Table 1.

$\mathrm{HCV}$ infection was proven by detecting circulating antiHCV antibodies (EIA-2 and RIBA-2, Ortho Diagnostic Systems, Raritan, NJ, USA) and HCVRNA (AMPLICOR HCV Test, v2.0. Roche Diagnostics, Alameda, CA, USA). HCV genotype was determined by a commercial, certified diagnostic test (VERSANT HCV Genotype 2.0, Siemens Healthcare Diagnostics, Deerfield, IL, USA). All the 
patients were HCV RNA positive since the ones who had undergone previous antiviral treatments were non-responders. In all these latter cases the therapy was stopped at least 12 months before the diagnosis of cancer and sample collection.

Liver disease was diagnosed according to standard, previously described criteria and was based on liver biopsy. The histopathology of liver samples was assessed by two independent expert pathologists external to the study, using the METAVIR algorithm [23].

HCC was diagnosed according to imaging techniques and/or biopsy [24].

A diagnosis of splenic marginal zone lymphoma (SMZL), nodal marginal zone lymphoma (NMZL) and DLBCL was made in nine, four and two NHL patients, respectively, according to the Revised European American Lymphoma Classification (REAL) [25].

\section{Methods}

Blood sampling was performed at the time of diagnosis of HCV-related chronic infection, NHL or HCC and before treatment. PBMCs were isolated from fresh anticoagulated blood by gradient precipitation on Lymphoprep (AxisShield PoC AS, Oslo, Norway) according to the manufacturer's instructions. After the second wash, the cells were counted and stored at $-80{ }^{\circ} \mathrm{C}$.

RNA extraction was performed using Trizol reagent (Invitrogen, USA) from $5 \times 10^{6}$ PBMCs according to the manufacturer's instructions. To monitor extraction efficiency, C. elegans miR-39 synthetic RNA oligonucleotide $\left(1.1 \times 10^{8}\right.$ copies $/ 10^{6}$ cells $)$ was added to PBMC samples and used as external control.

Reverse transcription was done using the TaqMan MicroRNA RT kit (Applied Biosystems, CA, USA) and
Table 1 Patient main demographical, laboratory and clinical data

\begin{tabular}{|c|c|c|c|c|}
\hline & HS $(n=40)$ & $\mathrm{HCV}(n=85)$ & NHL-HCV $(n=15)$ & HCC-HCV $(n=25)$ \\
\hline Mean age & $39 \pm 10.6$ & $54.2 \pm 11.9^{\mathrm{a}, \mathrm{b}, \mathrm{c}}$ & $62.5 \pm 7.23^{\mathrm{d}, \mathrm{e}}$ & $71.6 \pm 6.54^{\mathrm{f}}$ \\
\hline $\operatorname{Sex}(M / F)$ & $22 / 18$ & $60 / 25$ & $4 / 11^{\mathrm{g}, \mathrm{h}}$ & $16 / 9$ \\
\hline ALT (ULN) & $0.65 \pm 1.3$ & $1.75 \pm 1.26^{\mathrm{i}, 1}$ & $1.61 \pm 0.9^{\mathrm{m}, \mathrm{n}}$ & $3.86 \pm 2.5^{\circ}$ \\
\hline \multicolumn{5}{|l|}{ HCV genotype } \\
\hline 1 & & 55 & 8 & 18 \\
\hline 2 & & 15 & 4 & 4 \\
\hline 3 & & 13 & 2 & 3 \\
\hline 4 & & 2 & 1 & \\
\hline Viral titer (IU/ml) & - & $3.6 \pm 3.2$ & $3.3 \pm 2.8$ & $1.59 \pm 3.5^{\mathrm{p}}$ \\
\hline \multicolumn{5}{|l|}{ Histology } \\
\hline $\mathrm{F} 1 / \mathrm{F} 2$ & & 69 & 12 & - \\
\hline $\mathrm{F} 3 / \mathrm{F} 4$ & & 16 & 3 & 25 \\
\hline BCLC stage & - & - & - & \\
\hline A & & & & 16 \\
\hline B & & & & 2 \\
\hline $\mathrm{C}$ & & & & 2 \\
\hline nd & & & & 5 \\
\hline \multicolumn{5}{|l|}{ CLIP score } \\
\hline 0 & - & - & - & 12 \\
\hline 1 & & & & 5 \\
\hline 2 & & & & 2 \\
\hline 3 & & & & - \\
\hline 4 & & & & 1 \\
\hline nd & & & & 5 \\
\hline
\end{tabular}

Values are expressed as mean $\pm \mathrm{SD}$, unless otherwise indicated

$A L T$ alanine transaminase, $U L N$ upper level normal, $H S$ healthy subject, $H C V$ hepatitis $\mathrm{C}$ virus, $N H L$ nonHodgkin's lymphoma, HCC hepatocellular carcinoma, $I U$ international units

${ }^{\mathrm{a}} \mathrm{HCV}$ versus HS, $p<0.001 ;{ }^{\mathrm{b}} \mathrm{HCV}$ versus NHL-HCV, $p=0.01 ;{ }^{\mathrm{c}} \mathrm{HCV}$ versus $\mathrm{HCC}, p<0.001 ;{ }^{\mathrm{d}} \mathrm{NHL}$ versus HS, $p<0.001 ;{ }^{\mathrm{e}} \mathrm{NHL}$ versus HCC, $p<0.001 ;{ }^{\mathrm{f}} \mathrm{HCC}$ versus HS, $p<0.001 ;{ }^{\mathrm{g}} \mathrm{NHL}$ versus HCV, $p=0.001 ;{ }^{\mathrm{h}} \mathrm{NHL}$ versus HCC, $p=0.022 ;{ }^{\mathrm{i}} \mathrm{HCV}$ versus HS, $p<0.001 ;{ }^{1} \mathrm{HCV}$ versus $\mathrm{HCC}, p<0.001$; ${ }^{\mathrm{m}}$ NHL versus HS, $p=0.01 ;{ }^{\mathrm{n}} \mathrm{NHL}$ versus HCC, $p=0.006 ;{ }^{\circ} \mathrm{HCC}$ versus HS, $p<0.001 ;{ }^{\mathrm{p}}$ HCC versus $\mathrm{HCV}, p=0.008$ 
$160 \mathrm{ng}$ of total RNA. Expression levels of human miR-let7d, miR-16, miR-21, miR-26b, miR-146a, miR-155 and $C$. elegans miR-39 were evaluated by real-time PCR using specific TaqMan MicroRNA Assays (Applied Biosystems, CA, USA), according to the manufacturer's instructions.

Relative expression levels of the different miRNAs were evaluated with the $2^{-\Delta \mathrm{Ct}}$ method, using miR-let-7d as internal control to normalize miRNA expression levels, as previously described [22].

\section{Statistical analysis}

Data are expressed as mean \pm standard deviation (SD). Quantitative variables were analyzed using one-way analysis of variance (nonparametric ANOVA) or with the $t$ test for unpaired samples when appropriate. Categorical variables were analyzed with the $\chi^{2}$ test and Fisher's exact test when necessary. All tests were two-sided at a 0.05 significance level. Analyses were performed by Stata v.9.0 (StataCorpLP, College Station, TX, USA).

\section{Results}

The main demographical and clinical data of the patients are reported in Table 1.

As expected, patients in the NHL-HCV and $\mathrm{HCC}-\mathrm{HCV}$ groups were older than those in the HCV group ( $p=0.01$ and $p<0.001$, respectively), with more females in the NHL-HCV group (NHL-HCV vs. HCV, $p=0.001$ and NHL-HCV vs. HCC-HCV, $p=0.02$ ). There were no significant differences in terms of liver disease stage, alanine aminotransferase (ALT) serum levels or viremia titers between $\mathrm{HCV}$ and NHL-HCV patients, while in the HCCHCV group we observed higher ALT levels and lower $\mathrm{HCV}$ titers compared to the HCV group $(p<0.001$ and $p=0.008$, respectively).

There were no significant differences in HCV genotype distribution among the studied groups.

The expression levels of miR-146a were similar in all the tested groups, with no significant differences between patients and controls (Fig. 1).

MiR-16 and miR-155 showed a similar expression profile: significantly higher levels of these miRNAs were detected in the PBMCs isolated from NHL-HCV patients when compared to all other groups. We observed a strong upregulation of miR-16 in NHL-HCV groups when compared to HS, HCV and HCC-HCV ( $p<0.001$; Fig. 2a) and a higher expression of miR-155 in the PBMCs from NHL patients when compared to all the other groups $(p<0.001$ NHL-HCV vs. HS and vs. HCC-HCV; $p<0.01$ NHL$\mathrm{HCV}$ vs. HCV; Fig. 2b).
Interestingly, both miR-26b and miR-21 expression was altered in patients with $\mathrm{HCV}$-related malignancies. More specifically, a strong downregulation of miR-26b was detected in both the HCC and NHL groups when compared to the HCV and HS groups $(p<0.001$ and $p<0.05$, respectively; Fig. 3a), while miR-21 resulted in upregulation of the PBMCs from both NHL-HCV and HCC-HCV patients when compared to the HS and HCV groups (NHL$\mathrm{HCV}$ vs. HS, NHL vs. HCV, $p<0.001$; HCC-HCV vs. HS, HCC-HCV vs. HCV, $p<0.01$; Fig. 3b).

\section{Discussion}

In this study, we tested the modifications in the expression pattern of some miRNAs in PBMCs from patients with HCV-related HCC and NHL. We previously demonstrated that specific miRNA deregulation in PBMCs occurs in a smaller group of HCV-positive patients with NHL. Since interest in peripheral microRNAs as biomarkers is increasing and promising, we decided to test patients' serum and the results we obtained were often not reproducible. The main concern about the use of sera in our subjects was the lack of a reliable and stable miRNA working as endogenous control. For this reason, as an alternative choice, we tested the PBMCs, finding this kind of analysis trustworthy, consistent and easy to perform. Surprisingly, while performing the new experiments we observed interesting behavior of some miRNAs in the

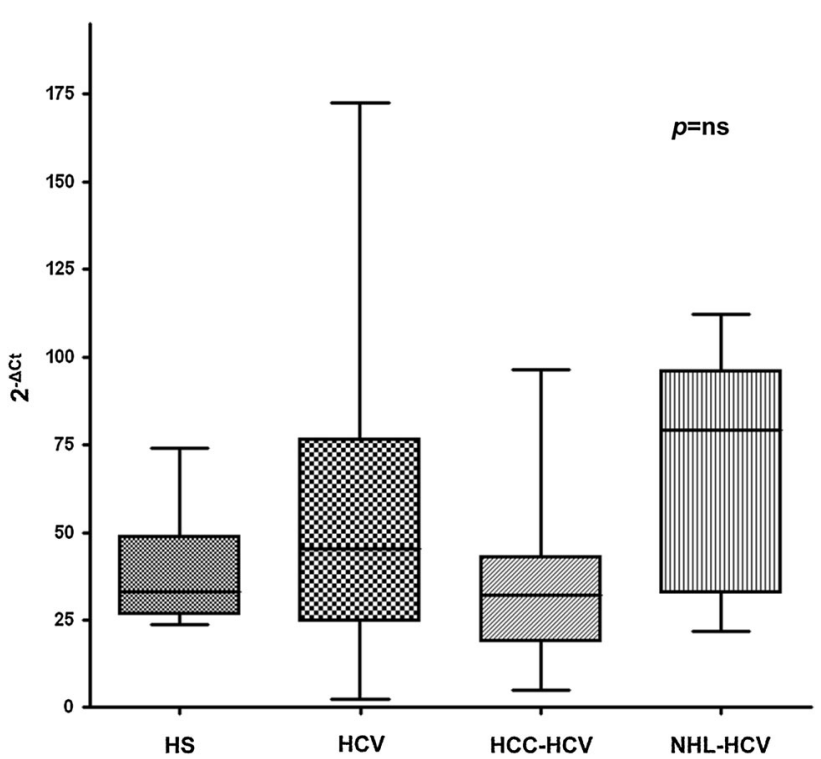

Fig. 1 Expression levels of miR-146a in healthy subjects (HS), HCV, NHL-HCV and HCC-HCV patients 

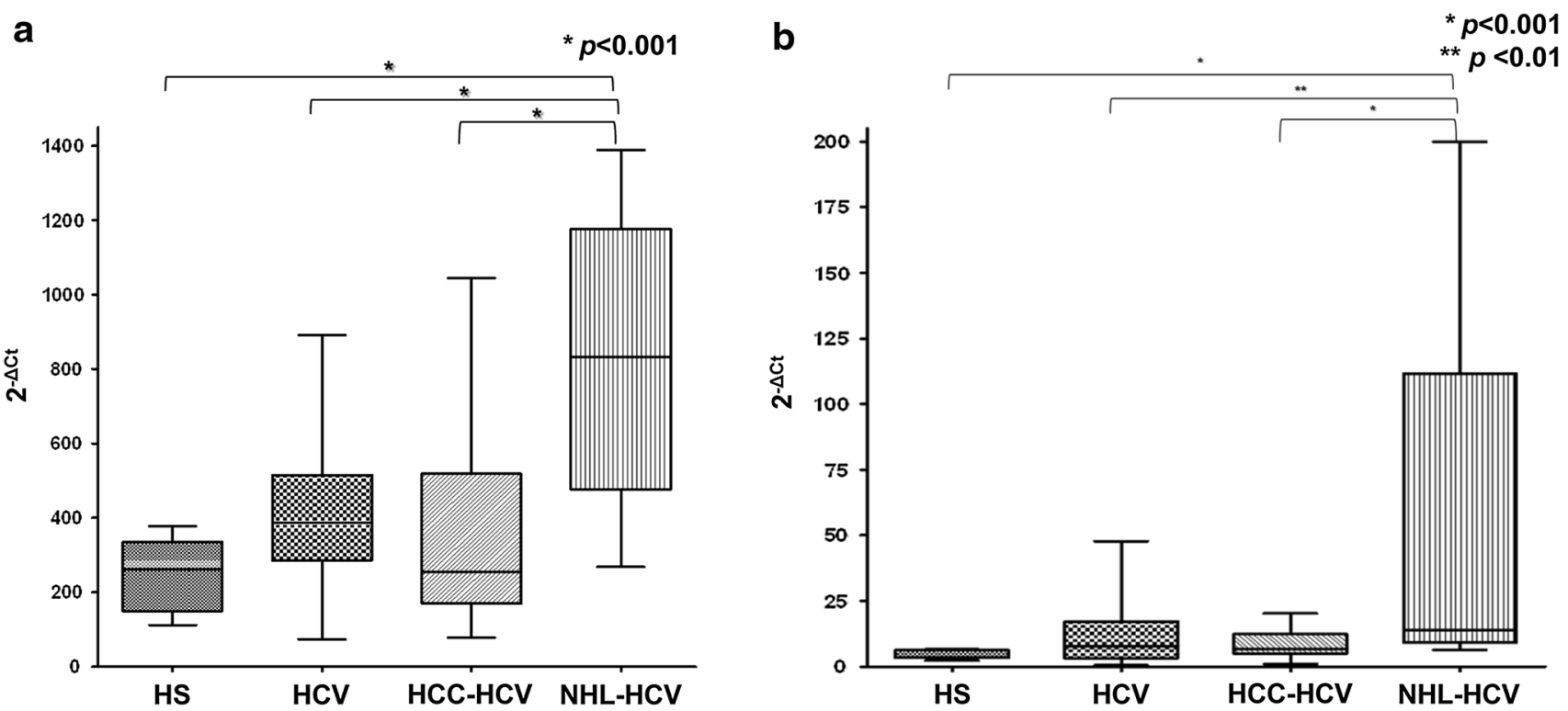

Fig. 2 Expression levels of miR-16 (a) and miR-155 (b) in healthy subjects (HS), HCV, NHL-HCV and HCC-HCV patients

population of $\mathrm{HCV}-\mathrm{HCC}$ patients we took as pathological controls.

We compared these results with data previously obtained in a group of HCV-positive NHL patients in order to identify markers of varying evolution for the two malignancies induced by this infection. We thereby selected and evaluated a panel of miRNAs shown to be involved in cancer. As previously described, miR-let-7d was used as internal control, being the most stable in our cohort of patients $[22,26]$.
We previously showed that miR-146a, a misregulated microRNA in several autoimmune/B-cell disorders, did not significantly differ in HCV-related lymphoproliferations compared to controls [22]. Here we report a similar profile in all the studied groups, including the HCC patients. These findings suggest that miR-146a is not involved in HCVrelated malignancies and/or altered by the presence of $\mathrm{HCV}$ infection.

MiR-16 and miR-155 showed a similar expression profile: significantly higher levels of these miRNAs were
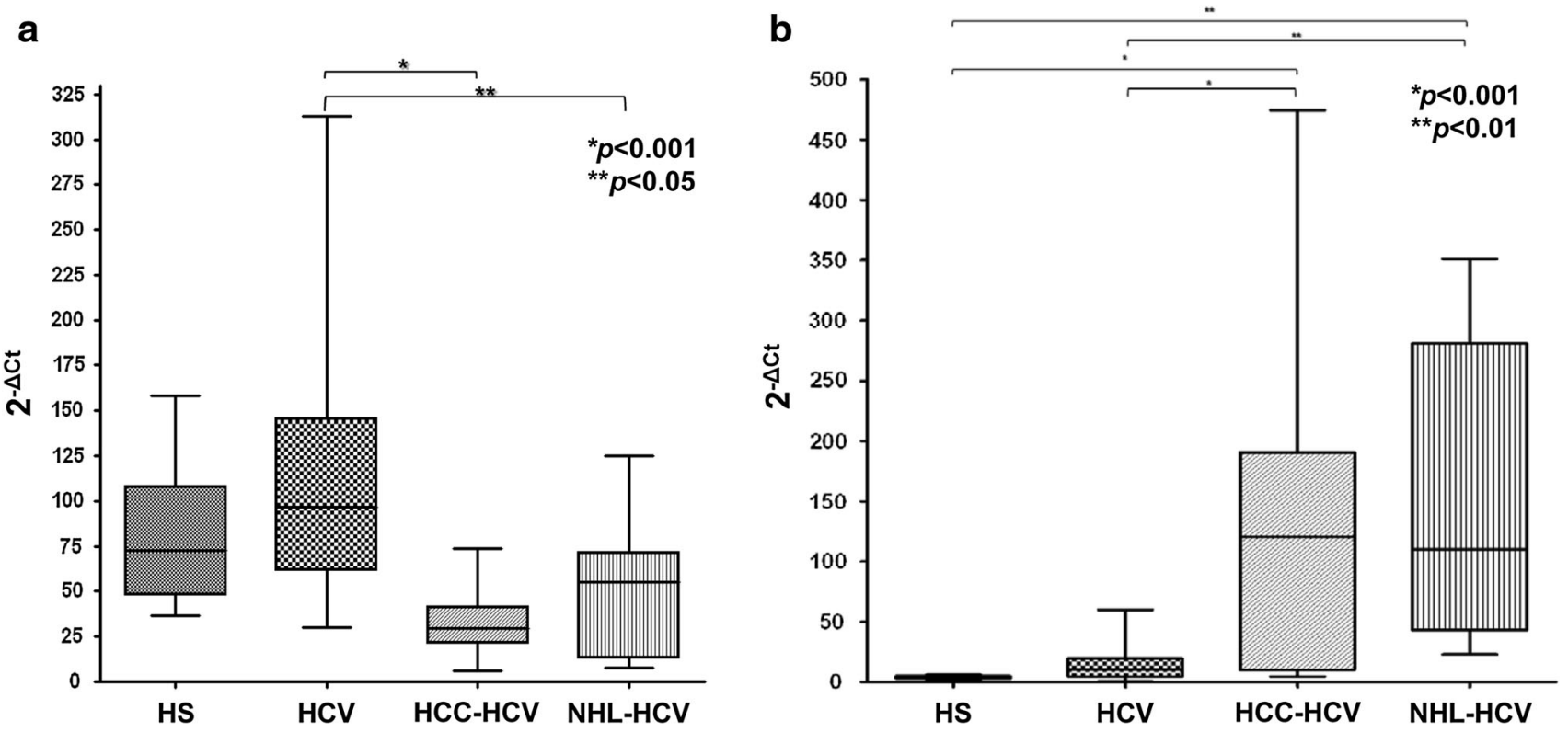

Fig. 3 Expression levels of miR-26b (a) and miR-21 (b) in HCV, NHL-HCV and HCC-HCV patients 
detected in the PBMCs isolated from NHL-HCV patients when compared to all other groups. Since these two miRNAs are upregulated in PBMCs of HCV-NHL patients, it is conceivable they could play a role as biomarkers of $\mathrm{HCV}$ related hematological malignancy.

Only two previous papers have speculated about the role of miR-16 expression in HCC: Qu et al. [27] reported significantly lower levels of this microRNA in the serum of HCC patients compared to controls, while Zeng et al. [28] attributed to it the ability of HCC to generate metastasis through repression of NOTCH signaling cascade. The data about the miR-16/HCC issue are scarce and conflicting, and we could not confirm any of the previously described scenarios since no aberrant expression of miR-16 was detected in our HCC cohort.

We were unable to attribute some significance to miR155 in HCV-related HCC but, as previously reported [22], its deregulation could indicate an evolution of $\mathrm{HCV}$ chronic infection towards B-cell malignancy. Interestingly, recent findings correlated miR-155 upregulation with the detection of the antigenomic strand of $\mathrm{HCV}$ in PBMCs [29]. Since the link between HCV infection of PBMCs and NHL was described almost 20 years ago [2], it is conceivable that the miRNA-155 upregulation we detected in HCV-related NHL, but not in HCC and control group PBMCs, may represent one of the mechanisms of virusinduced lymphomagenesis.

We noted similar behavior for miR-21 (upregulation) and miR-26b (downregulation) in both NHL and HCC, suggesting an involvement of these miRNAs in the infection-driven oncogenetic process and their role as peripheral markers for the two HCV-related cancers. MiR-21 was defined onco-miRNA since its levels were found to be increased in different malignancies, probably sharing one or more common pathways involving this specific miRNA in their pathogenesis [30, 31].

Elevated levels of miR-21 have been previously demonstrated in both HCC and lymphoma tumor tissue $[14,16]$. Studies conducted in vitro and on HCC tissues have shown miR-21 to be upregulated, and it was speculated that it decreases the expression of PTEN, a tumor suppressor, thus promoting cancer cell proliferation, migration and invasion [14]. A very recent study demonstrated that this microRNA increases T-lymphocyte survival in $\mathrm{T}$ cell acute lymphoblastic leukemia, mediating its function, in part via repression of the tumor suppressor programmed cell-death protein 4 (PDCD4) [32]; this could be another way through which miR-21 exerts its oncogenic power.

The increased expression of miR-21 in surgical and bioptic specimens of HCC has also been associated with tumor stage and poor prognosis, and miR-21 has emerged as an independent predictive factor of overall survival in
HCC patients [33]. The literature is rather scarce, and results are often controversial regarding circulating levels of this miRNA in HCCs of different etiologies [34-37]. On the other hand, only two papers have reported increased levels of miR-21 in serum/plasma samples from lymphoma patients (DLBCL and primary central nervous system lymphoma) [38, 39]. In this light, our findings of miR-21 upregulation in PBMCs from HCV-related HCC and NHL suggest a peripheral detection system more representative of the tissue tumor profile compared to serum/plasma analysis. The detection of microRNA levels in PBMCs could possibly reflect a more stable status compared to serum: the intracellular environment is certainly more confined than is serum, which is subjected to a non-stop systemic exchange of biological molecules. Furthermore, it is well known that cells circulating in peripheral blood maintain a resting status that makes this kind of model even more reliable for the stability of molecular expression patterns. The utility and accuracy of miRNA analysis in PBMCs has also been confirmed by previous studies [4042]. If confirmed in more numerous cohorts of patients, our observations about miR-21 in PBMCs could provide new perspectives for a reliable noninvasive test for both $\mathrm{HCV}$ related malignancies.

We observed a downregulation of miR-26b in HCV-associated NHL tissues [16], and we showed the same pattern in PBMCs isolated from this category of patients [19, 22]. Here we have confirmed previous results in a wider cohort of lymphoma patients, and, as explained above, driven by unexpected results in a small HCC group chosen as control, we tested a group of 25 patients with hepatic cancer and found statistically significant miR-26b downregulation.

Interestingly, recent studies have demonstrated that reduced expression of all the members of the miR-26 family (miR-26a-1, miR-26a-2, miR-26b) is correlated with a higher incidence of HCC and also with poor prognosis [21]. Since the miR-26 levels in liver are higher in females, these authors postulated a role as tumor suppressor for this miRNA, speculating that this could explain the varying susceptibility of males to develop HCC. The same authors showed a paradoxical improvement of overall survival in patients with lower miR-26 levels in the liver after adjuvant therapy with interferon- $\alpha$ [21, 43]; this finding clearly increases interest in identifying miR-26 as a peripheral biomarker not only of augmented risk and prognosis, but also as a predictor of therapy response.

The reasons for which the levels of some microRNAs in the PBMCs of HCC patients seem to reflect the expression pattern found in the liver tumor tissue are unknown. Further studies are needed to analyze PBMCs from surgery patients and tumor paired samples from HCC patients to understand the mechanisms underlying these miRNAs' deregulation. 
Regarding the HCV-positive HCC samples, we performed a statistical analysis to evaluate a possible correlation between miRNA levels and predicted survival terms obtained by CLIP score. Unfortunately, the number of patients included in our study did not allow reaching significance; therefore, further prospective and dedicated studies are needed to assess a relationship between miRNA levels and HCC prognosis.

This study strongly suggests that there is a real possibility of using the modifications in the miRNA expression pattern in PBMCs as potential markers of malignancy in chronically infected HCV patients. Furthermore, since specific miRNAs are differentially expressed in PBMCs from HCC and NHL patients, it is conceivable that these microRNAs could also be useful for understanding the evolution to HCV-related hepatic or hematological malignancies. Further prospective studies will help to confirm our results and reveal a translational application in clinical practice.

Acknowledgements We would like to thank Mary Forrest for critically reading and correcting the manuscript. This work was supported by grants from the "Associazione Italiana per la Ricerca sul Cancro" (AIRC) Investigator Grant \#1461, "Istituto Toscano Tumori" (ITT), "Ente Cassa di Risparmio di Firenze;" A.P. is supported by a 2015 fellowship "Fondazione Italiana per la Ricerca in Epatologia;" L.G. is supported by a 2015 fellowship "Fondazione Umberto Veronesi"; E.F. is supported by a 2015 AIRC ("Associazione Italiana per la Ricerca sul Cancro") fellowship.

\section{Compliance with ethical standards}

Conflict of interest Alessia Piluso, Laura Gragnani, Elisa Fognani, Elena Grandini, Monica Monti, Cristina Stasi, Elisabetta Loggi, Marzia Margotti, Fabio Conti, Pietro Andreone and Anna Linda Zignego declare that they have no conflict of interest.

Ethical statement All procedures followed have been approved by the local Ethics Committees and were in accordance with the Helsinki Declaration of 1975, as revised in 2008.

Informed consent All patients included in this study provided written informed consent in accordance with the principles of the Declaration of Helsinki, and the study was approved by the Ethics Committee of the University of Florence.

\section{References}

1. Jemal A, Bray F, Center MM, Ferlay J, Ward E, Forman D. Global cancer statistics. CA Cancer J Clin 2011;61:69-90

2. Zignego AL, Ferri C, Giannini C, La Civita L, Careccia G, Longombardo $\mathrm{G}$, et al. Hepatitis $\mathrm{C}$ virus infection in mixed cryoglobulinemia and B-cell non-Hodgkin's lymphoma: evidence for a pathogenetic role. Arch Virol 1997;142:545-555

3. McGivern DR, Lemon SM. Tumor suppressors, chromosomal instability, and hepatitis C virus-associated liver cancer. Annu Rev Pathol 2009;4:399-415

4. Peveling-Oberhag J, Arcaini L, Hansmann ML, Zeuzem S. Hepatitis C-associated B-cell non-Hodgkin lymphomas.
Epidemiology, molecular signature and clinical management. J Hepatol 2013;59:169-177

5. Ferri C, La Civita L, Longombardo G, Lombardini F, Pasero G, Zignego AL, et al. Hepatitis $\mathrm{C}$ virus in mixed cryoglobulinemia and B cell lymphoma [letter]. Clin Exp Rheumatol 1994;12:89-90

6. Zignego AL, Giannini C, Gragnani L. HCV and lymphoproliferation. Clin Dev Immunol 2012:980942

7. Zignego AL, Gragnani L, Giannini C, Laffi G. The hepatitis C virus infection as a systemic disease. Intern Emerg Med 2012;7(Suppl 3):S201-S208

8. Gragnani L, Fognani E, Piluso A, Zignego AL. Hepatitis C virusrelated mixed cryoglobulinemia: Is genetics to blame? World J Gastroenterol 2013;19:8910-8915

9. Di Leva G, Croce CM. miRNA profiling of cancer. Curr Opin Genet Dev 2013;23:3-11

10. Hou J, Lin L, Zhou W, Wang Z, Ding G, Dong Q, et al. Identification of miRNomes in human liver and hepatocellular carcinoma reveals miR-199a/b-3p as therapeutic target for hepatocellular carcinoma. Cancer Cell 2011;19:232-243

11. Giordano S, Columbano A. MicroRNAs: new tools for diagnosis, prognosis, and therapy in hepatocellular carcinoma? Hepatology 2013;57:840-847

12. Gramantieri L, Ferracin M, Fornari F, Veronese A, Sabbioni S, Liu CG, et al. Cyclin G1 is a target of miR-122a, a microRNA frequently down-regulated in human hepatocellular carcinoma. Cancer Res 2007;67:6092-6099

13. Wang XW, Heegaard NH, Orum H. MicroRNAs in liver disease. Gastroenterology 2012;142:1431-1443

14. Meng F, Henson R, Wehbe-Janek H, Ghoshal K, Jacob ST, Patel T. MicroRNA-21 regulates expression of the PTEN tumor suppressor gene in human hepatocellular cancer. Gastroenterology 2007; 133:647-658

15. Koberle V, Kronenberger B, Pleli T, Trojan J, Imelmann E, Peveling-Oberhag J, et al. Serum microRNA-1 and microRNA122 are prognostic markers in patients with hepatocellular carcinoma. Eur J Cancer 2013;49:3442-3449

16. Peveling-Oberhag J, Crisman G, Schmidt A, Doring C, Lucioni M, Arcaini L, et al. Dysregulation of global microRNA expression in splenic marginal zone lymphoma and influence of chronic hepatitis C virus infection. Leukemia 2012;26:1654-1662

17. Di Lisio L, Martinez N, Montes-Moreno S, Piris-Villaespesa M, Sanchez-Beato M, Piris MA. The role of miRNAs in the pathogenesis and diagnosis of B-cell lymphomas. Blood 2012;120:1782-1790

18. Bouteloup M, Verney A, Rachinel N, Callet-Bauchu E, Ffrench $\mathrm{M}$, Coiffier B, et al. MicroRNA expression profile in splenic marginal zone lymphoma. Br J Haematol 2012;156:279-281

19. Gragnani L, Fognani E, Piluso A, Zignego AL. Hepatitis C-associated B-cell non-Hodgkin lymphomas: the emerging role of miRNA-26b. J Hepatol 2013;59:1362-1363

20. Gramantieri L, Fornari F, Callegari E, Sabbioni S, Lanza G, Croce CM, et al. MicroRNA involvement in hepatocellular carcinoma. J Cell Mol Med 2008;12:2189-2204

21. Ji J, Shi J, Budhu A, Yu Z, Forgues M, Roessler S, et al. MicroRNA expression, survival, and response to interferon in liver cancer. N Engl J Med 2009;361:1437-1447

22. Fognani E, Giannini C, Piluso A, Gragnani L, Monti M, Caini P, et al. Role of microRNA profile modifications in hepatitis $\mathrm{C}$ virus-related mixed cryoglobulinemia. PLoS ONE 2013;8:e62965

23. Bedossa P, Poynard T. An algorithm for the grading of activity in chronic hepatitis C. The METAVIR Cooperative Study Group. Hepatology 1996;24:289-293

24. Bruix J, Sherman M. Management of hepatocellular carcinoma: an update. Hepatology 2011;53:1020-1022 
25. Harris NL, Jaffe ES, Stein H, Banks PM, Chan JK, Cleary ML, et al. A revised European-American classification of lymphoid neoplasms: a proposal from the International Lymphoma Study Group [see comments]. Blood 1994;84: 1361-1392

26. Qi R, Weiland M, Gao XH, Zhou L, Mi QS. Identification of endogenous normalizers for serum microRNAs by microarray profiling: U6 small nuclear RNA is not a reliable normalizer. Hepatology 2012;55:1640-1642; author reply 1642-1643

27. Qu KZ, Zhang K, Li H, Afdhal NH, Albitar M. Circulating microRNAs as biomarkers for hepatocellular carcinoma. J Clin Gastroenterol 2011;45:355-360

28. Zeng L, Yu J, Huang T, Jia H, Dong Q, He F, et al. Differential combinatorial regulatory network analysis related to venous metastasis of hepatocellular carcinoma. BMC Genom 2012;13(Suppl. 8):S14

29. Grek M, Piekarska A, Bartkowiak J, Fendler W, Kuydowicz J, Wroblewski P, et al. Coordinated increase of miRNA-155 and miRNA-196b expression correlates with the detection of the antigenomic strand of hepatitis $\mathrm{C}$ virus in peripheral blood mononuclear cells. Int J Mol Med 2011;28:875-880

30. Zhu W, Xu B. MicroRNA-21 identified as predictor of cancer outcome: a meta-analysis. PLoS ONE 2014;9:e103373

31. Wang Y, Gao X, Wei F, Zhang X, Yu J, et al. Diagnostic and prognostic value of circulating miR-21 for cancer: a systematic review and meta-analysis. Gene 2014;533:389-397

32. Junker F, Chabloz A, Koch U, Radtke F. Dicer1 imparts essential survival cues in Notch driven T-ALL via miR-21 mediated tumor suppressor Pdcd4 repression. Blood 2015. doi:10.1182/blood2014-12-61882

33. Karakatsanis A, Papaconstantinou I, Gazouli M, Lyberopoulou A, Polymeneas G, Voros D. Expression of microRNAs, miR-21, miR-31, miR-122, miR-145, miR-146a, miR-200c, miR-221, miR-222, and miR-223 in patients with hepatocellular carcinoma or intrahepatic cholangiocarcinoma and its prognostic significance. Mol Carcinog 2013;52:297-303
34. Bihrer V, Waidmann O, Friedrich-Rust M, Forestier N, Susser S, Haupenthal $J$, et al. Serum microRNA-21 as marker for necroinflammation in hepatitis $\mathrm{C}$ patients with and without hepatocellular carcinoma. PLoS ONE 2011;6:e26971

35. Xu J, Wu C, Che X, Wang L, Yu D, Zhang T, et al. Circulating microRNAs, miR-21, miR-122, and miR-223, in patients with hepatocellular carcinoma or chronic hepatitis. Mol Carcinog 2011;50:136-142

36. Zhou J, Yu L, Gao X, Hu J, Wang J, Dai Z, et al. Plasma microRNA panel to diagnose hepatitis B virus-related hepatocellular carcinoma. J Clin Oncol 2011;29:4781-4788

37. Tomimaru Y, Eguchi H, Nagano H, Wada H, Kobayashi S, Marubashi S, et al. Circulating microRNA-21 as a novel biomarker for hepatocellular carcinoma. J Hepatol 2012;56:167-175

38. Lawrie CH, Gal S, Dunlop HM, Pushkaran B, Liggins AP, Pulford K, et al. Detection of elevated levels of tumour-associated microRNAs in serum of patients with diffuse large B-cell lymphoma. Br J Haematol 2008;141:672-675

39. Mao X, Sun Y, Tang J. Serum miR-21 is a diagnostic and prognostic marker of primary central nervous system lymphoma. Neurol Sci 2014;35:233-238

40. Minutolo A, Conti B, Grelli S, Viscomi C, Labbadia G, Balsano C. Lymphocytes as liver damage mirror of HCV related adipogenesis deregulation. PLoS ONE 2014;9:e92343

41. Long L, Yu P, Liu Y, Wang S, Li R, Shi J, et al. Upregulated microRNA-155 expression in peripheral blood mononuclear cells and fibroblast-like synoviocytes in rheumatoid arthritis. Clin Dev Immunol 2013;2013:296139

42. Gupta P, Liu B, Wu JQ, Soriano V, Vispo E, Carroll AP, et al. Genome-wide mRNA and miRNA analysis of peripheral blood mononuclear cells (PBMC) reveals different miRNAs regulating HIV/HCV co-infection. Virology 2014;450-451:336-349

43. Ji J, Yu L, Yu Z, Forgues M, Uenishi T, Kubo S, et al. Development of a miR-26 companion diagnostic test for adjuvant interferon-alpha therapy in hepatocellular carcinoma. Int J Biol Sci 2013;9:303-312 